\title{
A Computer Vision Framework for Finger Tapping Evaluation in Parkinson's disease
}

Taha Khan', 2, Dag Nyholm ${ }^{3}$, Jerker Westin' and Mark Dougherty'

'Computer Engineering, School of Technology and Business Studies, Dalarna University, Falun, Sweden SE-78188

${ }^{2}$ School of Innovation, Design and Technology, Malardalen University, Vasteras, Sweden, SE-72123

${ }^{3}$ Department of Neuroscience, Neurology, Uppsala University, Uppsala, Sweden, SE-75185

DALARNA

UNIVERSITY

\section{BACKGROUND}

In the paced finger-tapping test (PFT), subjects attempt to reproduce a cer simul by pinching their index finger and thumb together with a (near) constant time interval. The varying amplitude in tapping and the series of inter-tap intervals are important clinical features for symptom assessment. Typically, the PFT is visually assessed in clinical practice. In order to avoid cost and effort of applying wearable sensors, a computer-vision method is introduced for non-invasive PFT evalvation.

\section{OBJECTIVE}

To define and evaluate a Computer-Vision (CV) method for scoring PFT in Parkinson's disease (PD) using quantitative motion analysis of indexfingers and to compare the obtained scores to the UPDRS (Unified Parkinson's disease Rating Scale) finger-taps (FT).

\section{METHODS}

A database consisting of 221 PFT videos from 6 PD patients was processed. The subjects were instructed to position their hands above their shoulders besides the face and tap the index-finger against the thumb consistently with speed. The subjects were facing towards a pivoted camera during recording. The videos were rated by two clinicians between the symptom severity levels '0: normal', '1: mild', '2: moderate' and '3: severe' using UPDRS-FT.

The CV method (figure 1) incorporates a motion analyzer [1] and a face detector [2]. The method first detects the face of testee in each video-frame (figure 2b). The frame is split into two images from facerectangle centre. Two regions of interest are located in each image to dect indexfinger motion of left and right hands respectively fligure 2c). The tracking of opening and closing phases (figure 3) of digure nant hand index-finger produces a tapping time-series (figure 4). This time-series is normalized by the face height (figure 2a). The normalizatime-series is normalized by the face height (figure $2 a$ ). The normalizavarying distance between camera and subject (i.e. farther the camera, lesser the amplitude).

Figure 1. System diagram of video-based Finger-Tapping Quantification

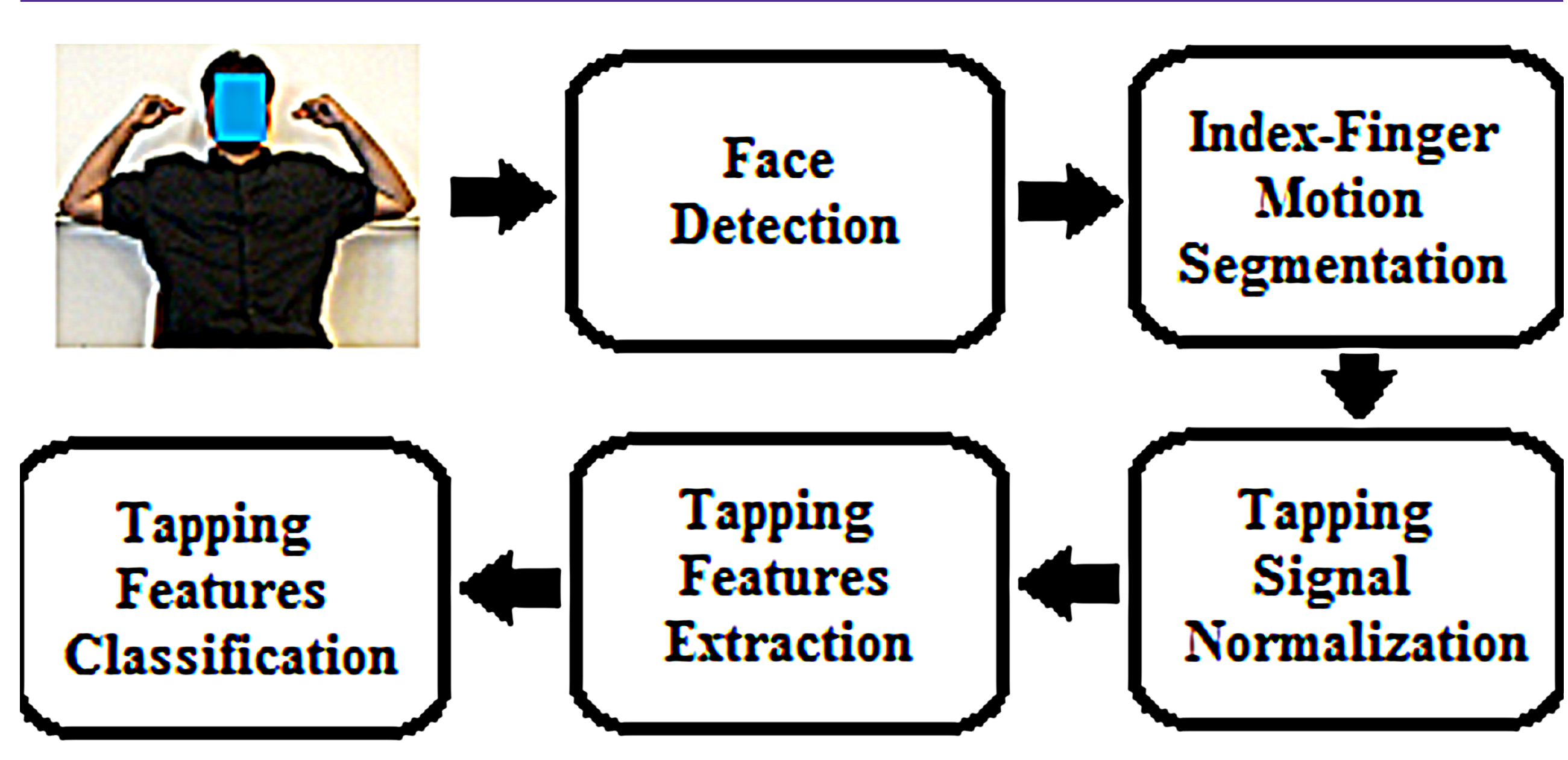

Figure 2. The Golden Ratio and the role of face detection in fingertapping quantification.

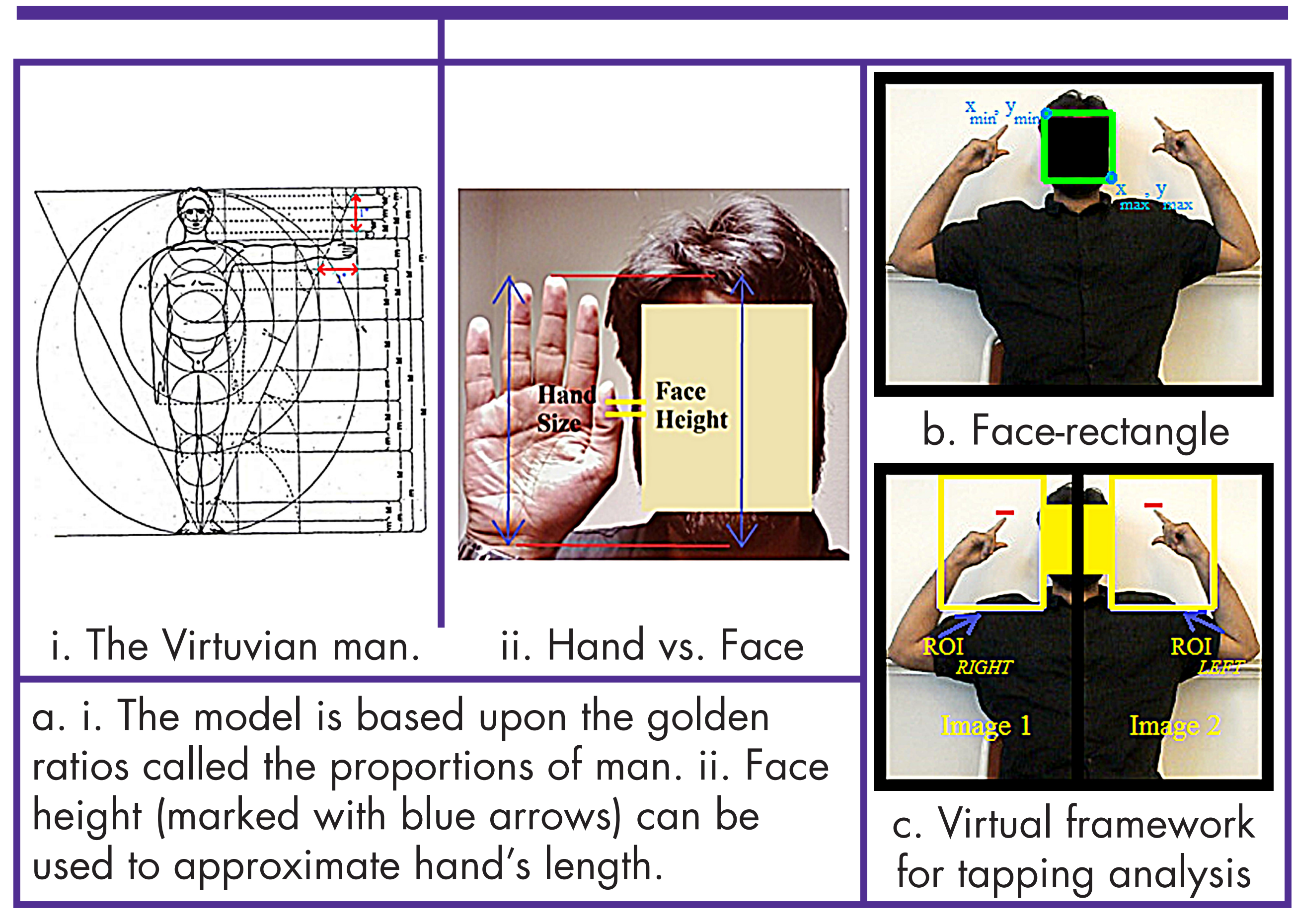

Figure 3. Index-fingers motion detection. Motion orientation of indexfingers in $\mathrm{ROI}_{\text {eff }}$ and $\mathrm{ROI}_{\text {right }}$ respectively is determined to identify the opening and closing phases of tapping. Tip of index-fingers are marked (red) to locate finger coordinates over time to produce a tapping signal.

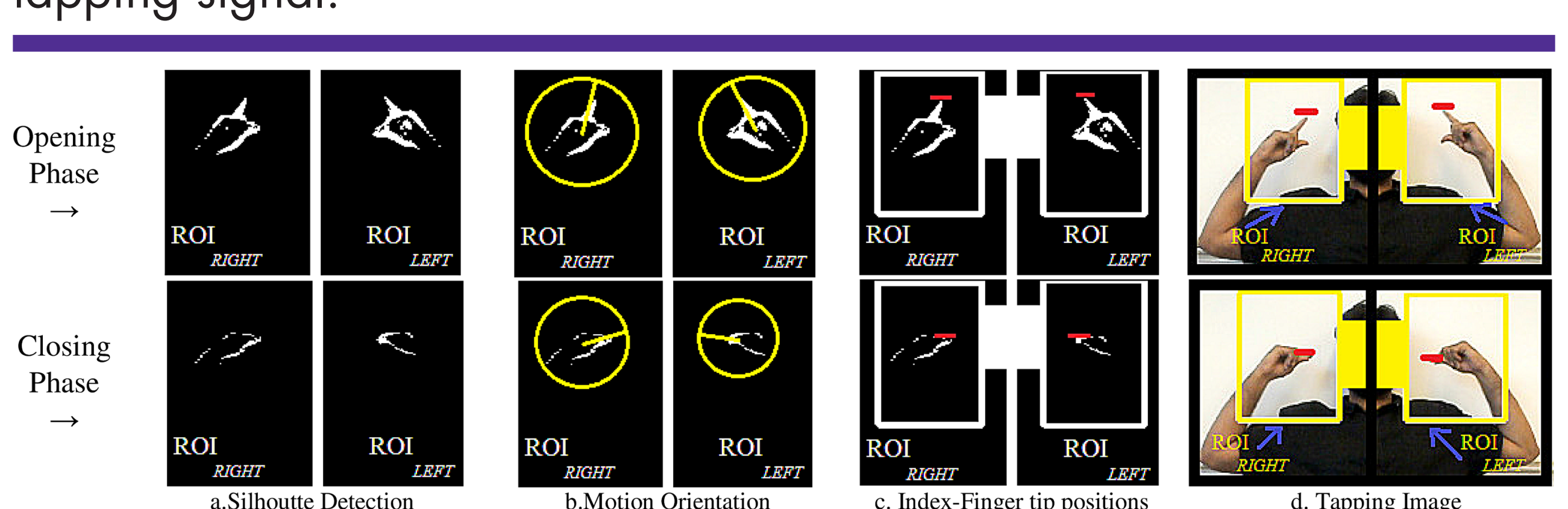

Figure 4. The tapping signals produced by the video processing f representative clinically rated samples. The tapping rhythm deteriorates as well as the amplitude decreases with the increase in symptom levels. Non-dominant (left) hand tapping signals deteriorates more than the dominant (right) hand.

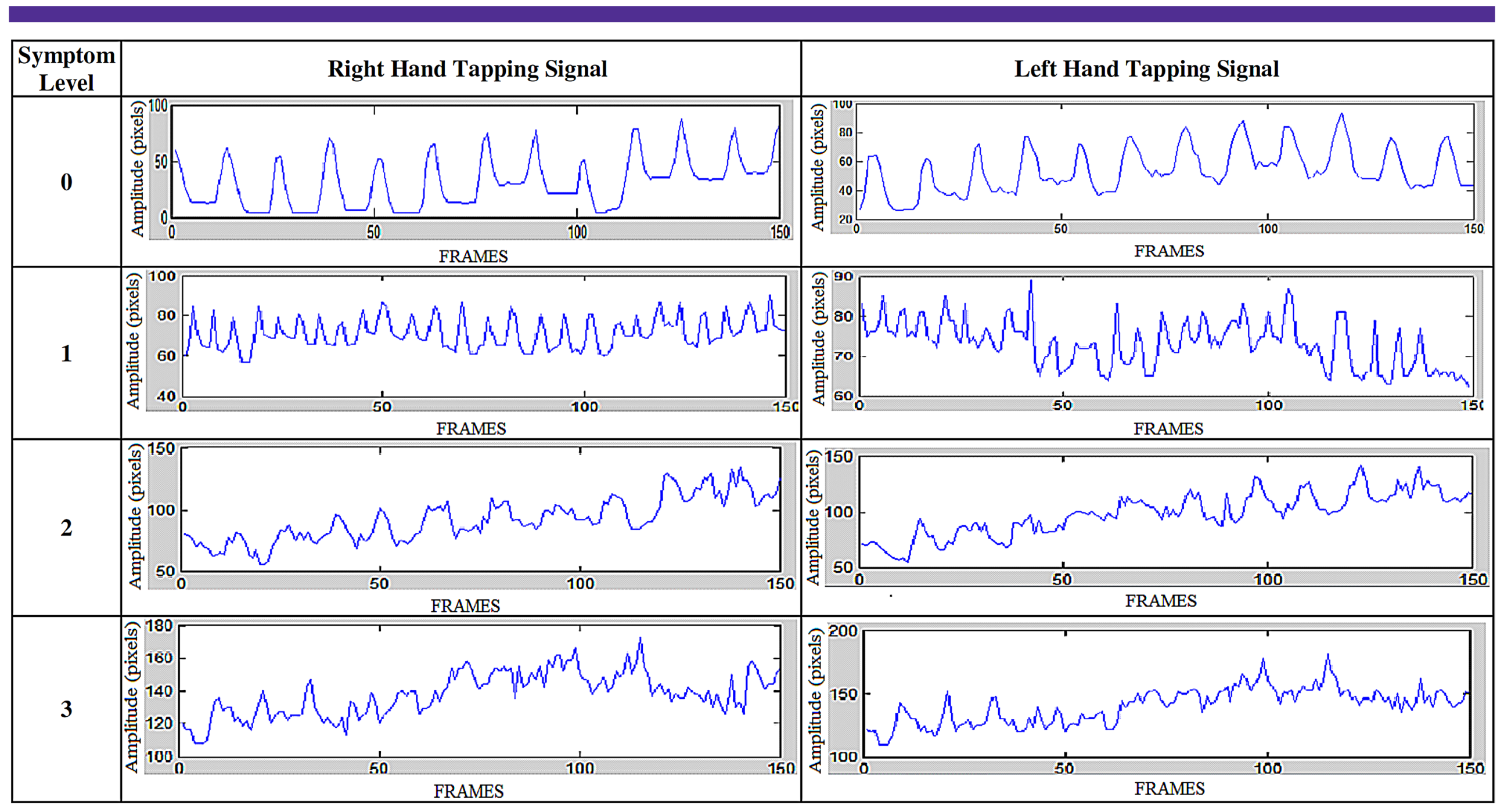

The target ratings provided by the two raters were averaged. A total of 15 features (table 1) were used for tapping quantification. Guttman correlation analysis [3] between the features and the averaged UPDRS FT ratings was performed. The strongly correlated features were selected and were used to classify the averaged UPDRS-FT levels using the K-nearest neighbor classifier (KNN) [4].

RESULTS AND DISCUSSION

The Guttman correlation coefficient ( 2 2) between PFT features and the averaged UPDRS-FT ratings was strong, i.e. $\mu 2$ was $0.51,-0.57$ $0.57,0.57,0.55,-0.55$ and 0.5 for Tn VTs, VA, VA, Tz, AvgCCNP and $F S$ respectively. A 10 -fold cross validation [5] in KNN classified the selected features between 3 UPDRS-FT levels with an accuracy of $78 \%$. An average area under the receiver operating characteristic curves of $82.6 \%$ supports feasibility of the obtained features to replicate clinical assessments. The classification results obtained from the KNN algorithm are shown in table 2. Each matrix row represents the actual class instances while each matrix column represents the instances in a predicted class. Matrix (numbers in bold) depict high true posiand ' 2.5 ' (86.3\%).

\section{CONCLUSIONS}

The system is able to track index-finger motion to estimate tapping symptoms in PD. It has certain advantages compared to other technologies (e.g. magnetic sensors, accelerometers etc.) for PFT evaluation to improve and automate the ratings. The uniqueness of this system is the utilization of facial features to normalize the tapping sign and to cope with camera calibration. Moreover, the method only requires a computer with a webcam. The subjects can perform the tapping task naturally in the same manner as instructed by movement tapping task naturally in the same manner as instructed by movemen disorder experts. An added advantage is that the subject's face in a
tapping video can be localized and blurred to avoid ethical issues in publishing and data sharing. The portability and ease of use makes it possible to perform the clinical assessments in the home environment.
REFERENCES

. G. R. Bradski, J.W. Davis, Motion segmentation and pose recognition with motion history gradients, Mach. Vision. Appl., 13 (2002) 174-184.

2. M.A. Turk, Face recognition using Eigenfaces, in Proc. IEEE CVPR 91, 1991, pp. 586-591.

3. L. Guttman, A Basis for Scaling Qualitative Data, AM SOCIOL REV, 9 (1944) 139-150.

4. P. Soucy, G.W. Mineau, A simple KNN algorithm for text categorization, in Proc. IEEE Int. conf. Data Mining, San Jose, CA USA, 2001, pp. 647-648

M. Stone, Cross-Validatory Choice and Assessment of Statistical Predictions, JSTOR, 36 (1974) 111-147.

Table 1. The Tapping Features. Features incorporate clinical symptoms (i.e. slow paced, amplitude reduction between index-finger and thumb, fatigue and arrhythmia) visually inspected by clinicians to rate between the UPDRS-FT levels.

\begin{tabular}{|c|c|c|}
\hline Sysmbol & Feature Description & 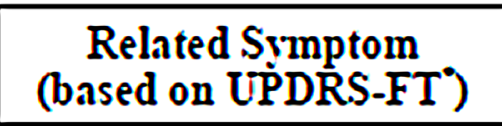 \\
\hline 1. $\mathrm{T}_{\mathrm{n}}$ & Total number of taps & Slowed Pace \\
\hline 2. $\Delta \mathrm{T}_{\mathrm{n}}$ & Difference between number of taps in ime slot 1 and 2. & Fatigue \\
\hline 3. $\mathrm{T}_{\mathrm{s}}$ & Tapping Speed & Slowed Pace \\
\hline 4. IT: & Variation Coefificient $(\mathrm{VC})$ in Tapping Speed & Fatigue \\
\hline 5. $\Delta . \mathrm{t}$ & Difiterence between the average maximum amplitude of finger taps in time slot 1 and 2 . & 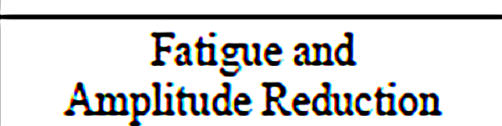 \\
\hline 6. $\triangle \mathrm{\Delta T}$ & Difference betrven the VCs in average maximum amplinde of inger taps in inime slot 1 and 2 . & $\begin{array}{c}\text { Fatiguve and } \\
\text { Amplitude Reduction }\end{array}$ \\
\hline 7.VA & VC in average maximum amplitude in finger taps & $\begin{array}{l}\text { Fatiguve and } \\
\text { Amplitude Reduction }\end{array}$ \\
\hline $8.0_{\mathrm{r}}$ & Average Opening Velocity of index-finger. & Slowed Pace \\
\hline 9. $C_{\mathrm{T}}$ & Average Closing Velocity of index-finger. & Slowed Pace \\
\hline 10.Ac & Tapping Acceleration. & Slowed Pace \\
\hline 11. $\mathrm{T}_{2}$ & Average Zero-Crossings & Slowed Pace \\
\hline 12. $T_{I}$ & Signal Energy. & $\begin{array}{c}\text { Slow wace and } \\
\text { Amplitide Reeuction }\end{array}$ \\
\hline 13. Argcasp & Mean of Cross-Corelation betrveen the Nomalized Peals. & Arrhythmia \\
\hline 14. Argecent & Mean of Cross.Corelation betrven the Normalized Valleys. & Arnyylumia \\
\hline 15.Fs & Standard Deviation of face-rectangle centroid during PFT. & - \\
\hline
\end{tabular}

Table 2. Classification Matrix. The matrix presents system performance based on a comparison between the averaged UPDRS FT ratings of tapping videos by the clinicians (y-axis) and the corresponding predicted UPDRS-ratings by the system (x-axis). The numbers in bold-italic depict a remarkable match between the system ratings and clinician rating

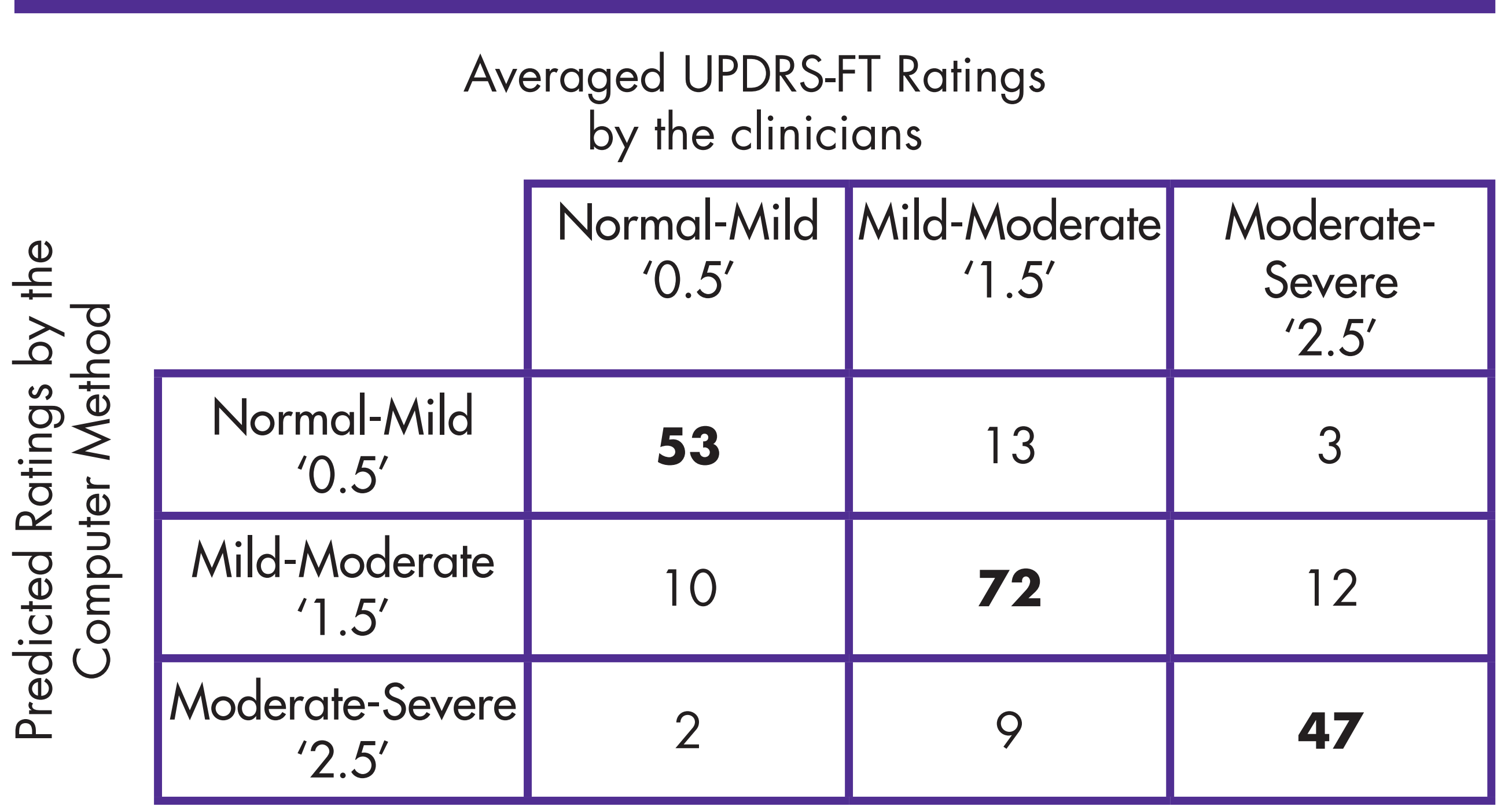

